\title{
FACTORS AFFECTING EXCLUSIVE BREASTFEEDING DURING THE FIRST SIX MONTHS IN
} KOREA

\author{
M. Kim ${ }^{1}$, Y.-M. Kim ${ }^{2}$, J.-H. Yoo ${ }^{1}$ \\ ${ }^{1}$ Department of Pediatrics, ${ }^{2}$ Department of Preventive Medicine, Dong-A University College of Medicine, \\ Busan, Republic of Korea
}

Objective: To identify the current status of exclusive breastfeeding (EBF) in Korea and analyze the factors affecting exclusive breastfeeding at six months of age.

Method: This study was based on the data obtained from the Korea National Health and Nutrition Examination Surveys (KNHANES) IV conducted from January 2007 to December 2008. A total 404 motherinfant pairs were recruited. In this study, exclusive breastfeeding was defined according to the criteria set forth by the World Health Organization (WHO).

Result: The rate of EBF was $60.9 \%$ for one month, $55.0 \%$ for three months, $35.4 \%$ for six months, $3.7 \%$ for nine months and $1.2 \%$ for twelve months after birth. According to a stepwise logistic regression analysis, factors that were positively associated with $\mathrm{EBF}$ at six months were: younger maternal age $(\mathrm{OR}=0.85,95 \%$ CI 0.79-0.92), higher maternal education level $(\mathrm{OR}=2.29,95 \%$ CI 1.17-4.46) and living in a capital area $(\mathrm{OR}=2.64,95 \%$ CI 1.46-4.75).

Conclusion: The rate of EBF in Korea is still suboptimal. To promote EBF, persistent and systematic education and campaigns regarding breastfeeding should be provided, particularly in vulnerable areas. 